\title{
An unusual case in endocrinology practice: Suture granuloma
}

\author{
${ }^{1}$ Cansu GB, ${ }^{1}$ Taskiran B, ${ }^{2}$ Bakar $\mathrm{T},{ }^{3}$ Cengiz BP \\ ${ }^{1}$ Division of Endocrinology and Metabolism, Yunusemre State Hospital, Eskisehir, Turkey; \\ ${ }^{2}$ Department of Radiology, Yunusemre State Hospital, Eskisehir, Turkey; \\ ${ }^{3}$ Department of Pathology, Yunusemre State Hospital, Eskisehir, Turkey \\ E-mail:bcansu74@hotmail.com
}

Objective. Suture granuloma, the rare complication of thyroidectomy, results from the use of nonabsorbable suture materials. Despite its typical ultrasound images and benign course, it carries utmost importance in the differential diagnosis of lymph nodes, recurrent nodules, and recurrence in the case of thyroid cancers.

Subject and Results. Fifty four years old female patient, who underwent bilateral thyroidectomy in July 2010, was diagnosed with multinodular goiter and incidentally discovered micropapillary carcinoma $(2 \mathrm{~mm})$. Four years later, she was readmitted to hospital due to painless swelling in the right and left anterior neck region. Ultrasonography revealed nodules with irregular boundaries, containing micro- and macro-calcifications and hyperechoic lines in both sides of the thyroid bed and isthmus. Fine needle aspiration biopsy was performed in the right and left sided mass and the cytological examination was compatible with the diagnosis of the suture granuloma.

Conclusions. Suture granuloma should be considered in the differential diagnosis of the local recurrence

Key words: suture granuloma, thyroidectomy, cytology

Thyroidectomy is a quite common surgical procedure worldwide. Suture granuloma, due to nonabsorbable sutures used, is an uncommon complication of thyroidectomy along with other common complications.

Despite its typical ultrasound images and benign course, it carries utmost importance in differential diagnosis of lymph nodes, recurrent nodules, and recurrence in case of thyroid cancers (Rettenbacher et al. 2001). We report a case that was considered as a recurrent nodular thyroid disease by US (ultrasonography), but it was confirmed to be a suture granuloma on a US-guided fine needle aspiration biopsy (FNAB).

\section{Subject and Results}

Fifty-four years old female was admitted to the hospital in December 2014 due to growth a mass in the right and left anterior neck regions for 6 months. She denied any pain or discomfort. She had a history of bilateral thyroidectomy in July 2010 with a pathologic diagnosis of multinodular goiter and incidentally discovered micropapillary carcinoma $(2 \mathrm{~mm})$. She did not receive radioactive iodine therapy afterwards and since then she has been on the levothyroxine replacement therapy. She did not undergo any imaging modality of the neck until swelling occurred on the anterior cervical region.

Physical examination revealed a $1.5 \times 2 \mathrm{~cm}$, welldefined, soft painless mass in the right thyroid bed and masses with similar features that measure $1 \times 1 \mathrm{~cm}$ in the isthmus and $1.5 \times 1 \mathrm{~cm}$ in the left thyroid bed. The masses did not move on swallowing. All the routine hematological and biochemical investigations were within normal limits. Thyroid function

Corresponding author: Guven Baris Cansu, MD., Yunusemre State Hospital, Division of Endocrinology and Metabolism, Eskişehir, TR-26190, Turkey; e-mail: bcansu74@hotmail.com. 
tests were normal, thyroid autoantibodies negative, and the thyroglobulin level was $2.4 \mathrm{ng} / \mathrm{ml}$.

The ultrasonography revealed hypoechoic lesions with irregular boundaries and containing micro and macrocalcifications and hyperechoic lines that measured $20.1 \times 13 \mathrm{~mm}$ in size on the right side and $16.5 \times 7.5 \mathrm{~mm}$ on the left side and $15.5 \times 9 \mathrm{~mm}$ in the isthmus (Figure 1). Increased vascularity was absent on Doppler ultrasound. Pathologic lymphadenopathy was not observed. The case was referred for a FNAB study.

Pathologic evaluation of FNAB yielded neutrophilic polymorphonuclear leucocytes, lymphocytes, and histiocytes in a dirty granular background of sparse colloid and foreign-body multinucleated giant cells within intense inflammatory cell infiltrate forming cell debris in almost every region (Figures 2 and 3). Hence, these features were those of a suture granuloma.

\section{Discussion}

Suture granuloma is due to nonabsorbable suture materials used during surgery of anywhere in the body. As an infrequent complication of thyroidectomy, it may be confused with tumor recurrence in the case of thyroid cancer and recurrent nodules in case of nodular goiter. Therefore, differential diagnosis should be done with meticulous attention.

Suture granuloma probably results from a chronic inflammatory process. The pathogenesis involves two steps. The first step is the initial reaction of the tissue, which reflects the amount of injury inflicted by the passage of the needle. After the initial reaction subsides, the second step occurs. In this step, the suture material causes specific inflammatory reactions (Postlethwait et al. 1975). Histologically, it is a benign granulomatous inflammatory lesion characterized by

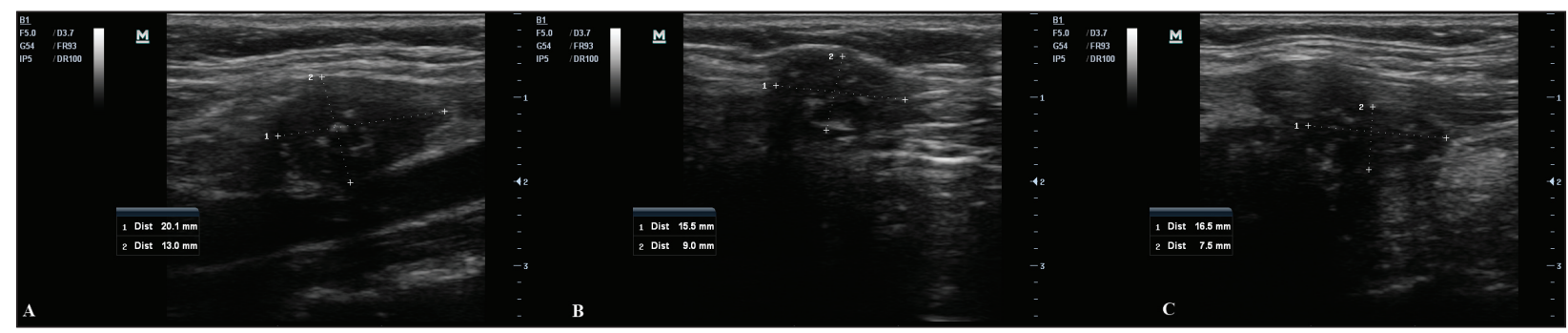

Figure 1. (A) Transverse section on ultrasound. A $20.1 \times 13 \mathrm{~mm}$, well-defined, hypoechoic lesion containing hyperechoic lines in the right thyroid bed. (B) Transverse section on ultrasound. A $16.5 \times 7.5 \mathrm{~mm}$, well-defined, hypoechoic lesion containing hyperechoic lines, and microcalcification foci in the left thyroid bed. (C) Transverse section on ultrasound. A $15.5 \times 9 \mathrm{~mm}$, well-defined, hypoechoic lesion containing hyperechoic lines, and microcalcification foci in the isthmus thyroid bed.

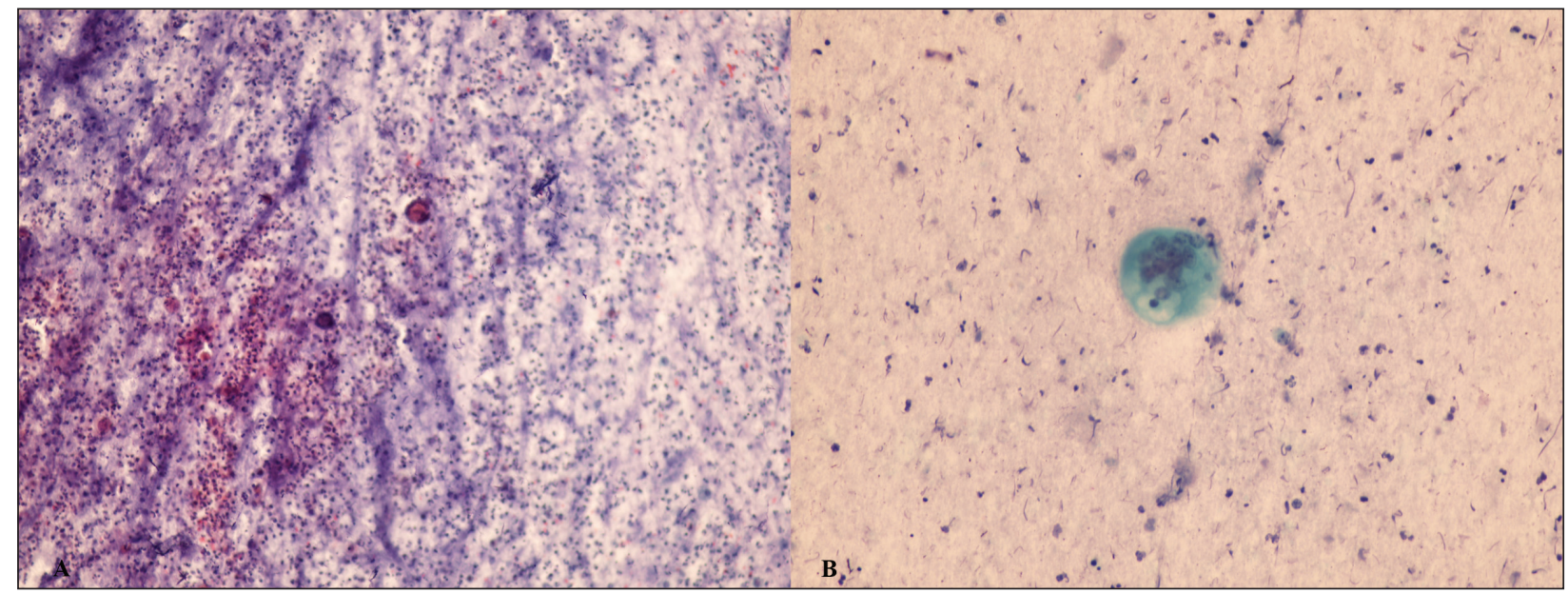

Figure 2. (A) Foreign-body multinucleated giant cells within cell debris and intense inflammatory cell infiltrate composed of neutrophilic polymorphonuclear leucocytes, lymphocytes, and histiocytes in background (PAP, $\times 100)$. (B) Multinucleated giant cells in a dirty granular background of sparse colloid (PAP, $\times 400)$. 
a histiocytic reaction with foreign-body giant cells around the sutures (Helms and Clark 1977; Pearl and Someren 1980).

At the beginning, classical features of inflammatory reaction, including erythema, swelling, and pain, occur and as they subside the patients are frequently admitted to hospitals due to complaints of painless and progressively growing solid masses.

Our patient was admitted to our clinic due to painless cervical mass growing progressively in the preceding months as well.

Suture granuloma can be confused with residual thyroid tissue in case of thyroid carcinoma, especially in non-RAI receivers and thyroglobulin level does not help in such cases. Therefore, other diagnostic modalities should be used. Eldridge et al. (1987) have claimed that since suture granuloma occurs within two years of thyroidectomy, this time interval can be used for diagnosis. However, other investigators have reported a time span from a few months to several years (Rettenbacher et al. 2001). Therefore, it may be assumed that the time interval between the operation and formation of suture granuloma is not a reliable feature for differential diagnosis. In our patient swelling in the throat was defined four years after thyroidectomy.

Other diagnostic modalities are imaging methods. Rettenbacher et al. (2001) have defined the typi- cal features of the suture granuloma on the ultrasound. Hypoechoic lesion with hyperechoic double lines (rail-like lines) and/or a single line strongly suggest suture granuloma. In our case, there were characteristic US findings of suture granulomas in the right thyroid bed. But such findings were absent on the left side. Nevertheless, ultrasonographic features, suggesting malignancy (marked hypoechoic echogenicity, microcalcification, taller than wide in shape, blurred margin, irregular shape, and intranodular blood flow in Doppler sonography) can also be observed in the suture granuloma (Chung et al. 2006). FNAB is of utmost importance in correct diagnosis. Multinucleated giant cells, dense histiocytes, and inflammatory cells, indicating foreign body reaction, constitute the typical cytological picture of the suture granuloma and confirms the diagnosis.

The suture granuloma should be considered in differential diagnosis of local recurrence of the disease in thyroid bed along with the lymphadenopathy and tumor recurrence in patients who underwent thyroidectomy and have ultrasound images suggesting recurrence. Diagnosis can be established by FNAB avoiding unnecessary imaging modalities such as MR or PET CT. Histiocytic reaction with foreignbody multinucleated giant cells on cytological evaluation confirms the diagnosis.

\section{References}

Chung YE, Kim EK, Kim MJ, Yun M, Hong SW. Suture granuloma mimicking recurrent thyroid carcinoma on ultrasonography. Yonsei Med J 47, 748-751, 2006.

Eldridge PR, Wheeler MH. Stitch granulomata after thyroid surgery. Br J Surg 74, 62, 1987.

Helms CA, Clark RE. Post-herniorrhaphy suture granuloma simulating a bladder neoplasm. Radiology 124, $56,1977$.

Pearl GS, Someren A. Suture granuloma simulating bladder neoplasm. Urology 15, 304-306, 1980.

Postlethwait RW, Willigan DA, Ulin AW. Human tissue reaction to sutures. Ann Surg 181, 144-150, 1975.

Rettenbacher T, Macheiner P, Hollerweger A, Gritzmann N, Weismann C, Todoroff B. Suture granulomas: sonography enables a correct preoperative diagnosis. Ultrasound Med Biol 27, 343-350, 2001. 